LBL-33287

HIFAN-572

\title{
Induction Accelerator Development for Heavy Ion Fusion
}

\author{
L. L. Reginato \\ Accelerator \& Fusion Research Division \\ Lawrence Berkeley Laboratory \\ University of California \\ Berkeley, California 94720
}

Submitted to the 1993 Particle Accelerator Conference

Washington, D. C., May 17 - 20, 1993

*This work was supported by the Office of Energy Research, Office of Fusion Energy, U. S. Department of Energy under Contract No. DE-AC03-76SF00098. 


\title{
Induction Accelerator Development for Heavy Ion Fusion*
}

\author{
Louis I. Reginato for the HIFAR EngineeringGroup** \\ University of California Lawrence Berkeley Laboratory \\ 1 Cyclotron Road, MS 47-112, Berkeley, CA 94720 USA
}

\begin{abstract}
For approximately a decade, the Heavy Ion Fusion Accelerator Research (HIFAR) group at LBL has been exploring the use of induction accelerators with multiple beams as the driver for inertial fusion targets. Scaled experiments have investigated the transport of space charge dominated beams (SBTE), and the current amplification and transverse emittance control in induction linacs (MBE-4) with very encouraging results. In order to study many of the beam manipulations required by a driver and to further develop economically competitive technclogy, a proposal has been made in partnership with LLNL to build a $10 \mathrm{MeV}$ accelerator and to conduct a series of experiments collectively called the Induction Linac System Experiments (ILSE).

The major components critical to the ILSE accelerator are currently under development. We have constructed a full scale induction module and we have tested a number of amorphous magnetic materials developed by Allied Signal to establish an overall optimal design. The electric and magnetic quadrupoles critical to the transport and focusing of heavy ion beams are also under development. The hardware is intended to be economically competitive for a driver without sacrificing any of the physics or perfornance requirements.

This paper will concentrate on the recent developments and tests of the major components required by the ILSE accelerator.
\end{abstract}

\section{INTRODUCTION}

The LBL approach to a HIF driver using multiple beams in one induction accelerator and combining them into fewer beams has many unique advantages, such as bigh repetition rate, reliability, high efficiency, and electromagnetic focusing which allow long stand-off from the target. To further investigate the feasibility of this approach beyond MBE-4, the HIFAR group has proposed a series of scaled experiments called the Induction Linac Systems Experiments (ILSE) [1]. In ILSE we will not only investigate beam transport physics but will also develop accelerator technology and a capital cost database which will confirm the technological soundness and economics of this approach to HIF.

In ILSE, four potassium beams will be accelerated from a $2 \mathrm{MeV}$ injector to $5 \mathrm{MeV}$ by the electrostatic focusing section, combined into a single beam, and accelerated to 10 $\mathrm{MeV}$ by the magnetic focusing section. The line charge density will be the same as for a full scale driver, $0.25 \mu \mathrm{C} / \mathrm{m}$, and charge compression or current amplification occurs continuously throughout the accelerator. To maintain iongitudinal stability, the acceleration waveforms are also

\footnotetext{
* Work supported by the Director, Office of Energy Research, Office of Fusion Energy, U.S. Department of Energy under Conract No. DE-AC03-76SF00098.

${ }^{* *} A$ list of the contributors is listed under acknowlegements.
}

continuously changing throughout the accelerator; initially the acceleration waveforms are ramped or trapezoidal with $1.5 \mu \mathrm{s}$ duration while the final stages will have square waveforms with 400 ns duration. To transport the four space charge dominated beams, electrostatic quadrupoles with a half lattice period of $33 . \mathrm{cm}$ and $41 \mathrm{~cm}$ will be used. After the beams reach the $5 \mathrm{MeV}$ acceleration, a four-to-one beam combiner will be used to inject a single beam into the magnetic quadrupole transport section. This section with a balf lattice period of $50 \mathrm{~cm}$ will accelerate the ions from $5 \mathrm{MeV}$ to 10 $\mathrm{MeV}$. This paper will describe the accelerator design and the technology development.

\section{MAGNETIC MATERIALS}

The induction accelerator is inherently a very low gradient $(<1 \mathrm{MeV} / \mathrm{m}$ ) but very high current device (10s of $\mathrm{kA}$ ). The drive system for an induction linac is typically a distributed or a lumped pulse forming network with the proper impedance to supply both the beam current $\left(I_{B}\right)$ and the core inagnetizing current $\left(I_{M}\right)$ at the desired voltage. The efficiency is simply the ratio $I_{B} /\left(I_{B}+I_{M}\right)$. For an accelerator such as the ATA where the magnetizing current was $800 \mathrm{~A}$ and the beam current $8 \mathrm{kA}$, the efficiency is very high. In a driver for inertial confinement fusion, a very large fraction of the induction linac will be required to accelerate many kiloamperes of beam. The induction cores will no doubt be large in diameter and likewise require several kiloamperes of magnetizing current, making this approach to acceleration reasonably efficient ( $>50 \%$ ). The front end of a driver, prior to large charge compression or current multiplication, will be accelerating tens to hundreds of amperes which will make the efficiency quite small $(<10 \%)$. ILSE is basically the front end of driver and in order to optimize the design, a magnetic material should be chosen with a maximum flux swing $(\Delta B)$ and a minimum magnetization current $(\Delta H)$. The ferromagnetic material is, therefore, one of the most important components of an induction linac because it seis the requirements for the acceleration waveform drive system. Historically, short pulse (< $100 \mathrm{~ns}$ ) induction accelerators have utilized ferrite as the magnetic material of choice, whereas those for longer pulses have used nickel-iron and amorphous materials

The voltage gradient and the pulse duration will establish the volt-seconds required by the cells. For ILSE, a voltage gradient of 300 to $400 \mathrm{kV} / \mathrm{m}$ and a pulse duration of one to two microseconds will require cores of one to two meters in diameter. It is imperative to find a material with the maximum flux swing and the minimum magnetizing current. A significant effort has already been invested in the investigation of the magnetic materials which are appropriate for this application [2] There are several materials which satisfy the technical requ. - ments for ILSE. The conventional $\mathrm{Ni}-\mathrm{Fe}$ alloys that have been used for many decades and the 
relatively new amorphous materials (Metglas* ) satisfy these requirements. The choice of materials is therefore based on economic advantages. Recent investigation shows that the Metglas alloys hold an advantage over the conventional $\mathrm{Ni}-\mathrm{Fe}$ alloys. The Allied Metglas amorphous material 2605 series can be mass produced as a thin ribbon $(15-20 \mu \mathrm{m})$ with high resistivity and practically any width. This material is used extensively by the $60 \mathrm{~Hz}$ power industry. For this application, annealing is essential in order to achieve maximum flux swing and minimum loss. Annealing is typically done after the ribbon is wound into a core without interlaminar insulation. Interlaminar insulation is not required for the $60 \mathrm{~Hz}$. application because the volts per layer of ribbon are very small $(\mathrm{mV})$. For the ILSE application where the iates of magnetization are between 2-6 Tesla/ $\mu$ s and 10-20 V per layer can be generated, some insulation will be required to maintain low eddy current losses. Previous efforts have concentrated on finding interlaminar insulation which allows annealing after the material is wound into a core. Annealing embrittles the material, and winding a core afterwards is not practical. After several years of effort, insulators were found which beld off sufficient volts per layer and that could withstand annealing temperatures without degradation of the magnetic properties. Application of those coating, however, resulted in a considerable cost increase for the finished core. Clearly, the most cost effective option for ILSE is to use Metglas as-cast (unannealed) and wound with thin mylar insulation $(2.5 \mu \mathrm{m})$ to achieve turn-to-turn insulation and a high packing factor. Experimental data [3] has shown that at high magnetization rates, magnetic losses can be described fairly closely by saturation wave theory. In this mudel, unlike the $60 \mathrm{~Hz}$ case where hysterisis losses are dominant, the losses depend on the square of the ribbon thickness, the rate of magnetization, and the inverse of the resistivity. Extensive testing and comparison between annealed and unannealed Metglas showed that for the latter there is slight rounding of the B-H loop, but the flux swing and the magnetization losses are nearly the same (Fig.1). A number of alloys were tested (Fig.2) and it appears that the unannealed $2605 \mathrm{SC}$ material is optimal for IISE. The flux swing is $2.5-3$ Tesla, the losses with $1 \mu \mathrm{s}$ saturation times are about 1000 Joules $/ \mathrm{m}^{3}$, and the packing factor with $2.5 \mu \mathrm{m}$ milar is greater than $80 \%$.

The recent conceptual design report (CDR) used this material for our baseline cost estimates. Studies of other magnetic materials such as the Metglas TCA and the conventional $\mathrm{Ni}-\mathrm{Fe}$ is continuing, and a final decision on the most cost effective material will not be made until after the project is approved.

\section{A. Induction Cell}

The principle of linear acceleration by magnetic induction has been thoroughly covered in many publications. Heavy ion induction accelerators, however, are unique in their requirements. The acceleration waveforms are continuously variable throughout the ILSE acceleration cycle and the focusing half lattice periods (HLP) varies accordingly from an initial $33 \mathrm{~cm}$ for the first 16 modules to $41 \mathrm{~cm}$ for the next 16 and finally $50 \mathrm{~cm}$ for the remainder of the accelerator. A

\footnotetext{
* Metglas is a trademark of Allied Signal Corporation.
}
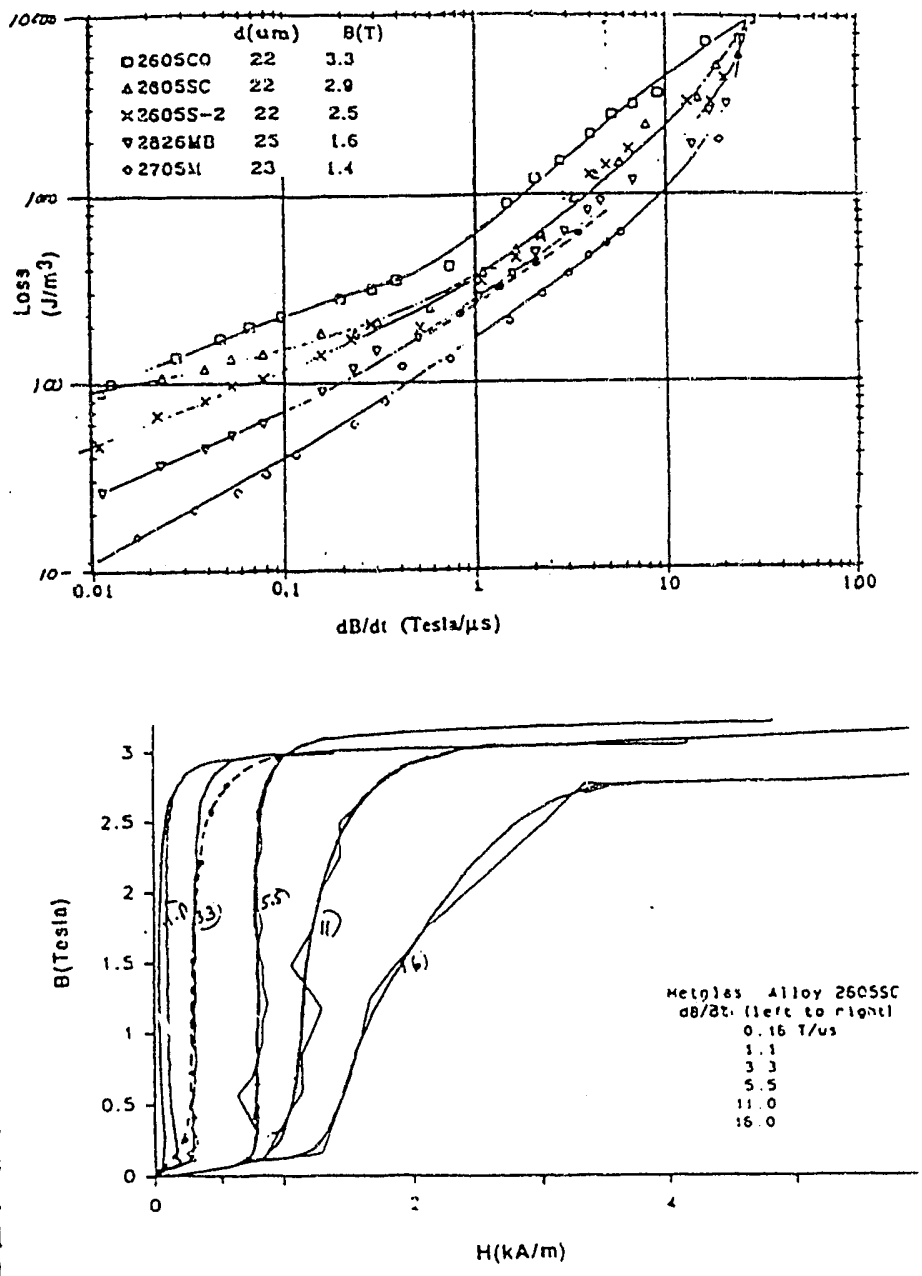

Figure 1. Top: Losses $\left(\mathrm{J} / \mathrm{m}^{3}\right)$ at different rates of magnetization $\mathrm{dB} / \mathrm{dt}(\mathrm{T} / \mu \mathrm{s})$ measured by Allied for annealed

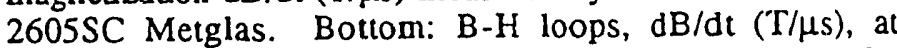
different rates of magnetization, measured by Allied, for annealed 2605SC Metglas. The dotted lines represent LBL data for the same material unannealed with $2.5 \mu \mathrm{m}$ of Mylar wound between curns.

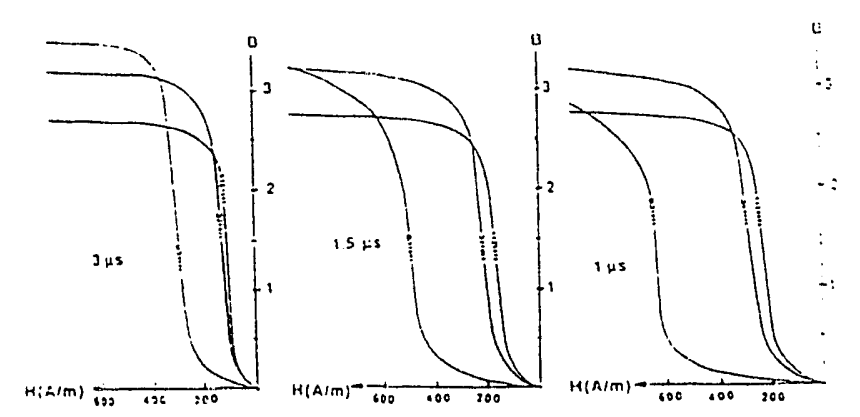

Figure 2. Dynamic magnetization curves taken at constant voltage and different saturation times for unannealed 2605 alloys. 
number of factors influenced our decision to choose the 2"

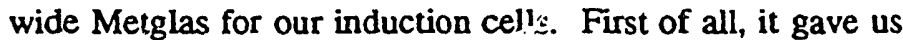
the flexibility to use identical cells for the different half lattice periods. For example, the $33 \mathrm{~cm}$ HLP uses three cells, the 41 $\mathrm{cm}$ four cells and the $50 \mathrm{~cm}$ five cells bolted together to form a module. Between each module there is axial space for high voltage feedthrough, magnet power, and most importantly, alignment and structural supports for the quadrupoles. $A$ second reason for the $2^{\text {" }}$ width was the fact that we wanted to avoid cumbersome liquid insulation (oil) in the acceleration gap. With the 2" width, the voltage per gap is $33 \mathrm{kV}$ which can be held off reliably with gas insulation $\mathrm{SF}_{6}$ or $\mathrm{CO}_{2}+$ $\mathrm{N}_{2}$ ). Another argument for maintaining acceleration voltages of $33 \mathrm{kV}$ is that the line modulators which drive the core can utilize reliable and inexpensive glass thyratrons rather than expensive high voltage ceramic units or unreliable spark gaps. Another advantage of using a narrower ribbon is that the voltage per layer is lower, thus allowing a thinner interlaminar insulation $(2.5 \mu \mathrm{m})$ to hold off the voltage, and this results in a higher packing factor $(0.8$ to 0.9$)$. The inside diameter of the induction cell is determined by the size of four beam electrostatic quadrupole and the voltage hold off requirements. The gradient and pulse duration now dictate the volt-seconds, hence the outside diameter. Allowing 50\% more volt-seconds than the minimum dictated by the $1 \mu \mathrm{s}$ pulse, the outside diameter is $1.5 \mathrm{~m}$. The magnetic field $\mathrm{H}$ is related 10 magnetizing current $\mathrm{I}$ by $\mathrm{H}=\mathrm{I} / 2 \pi \mathrm{r}$. Because the outside radius is about three times the inside radius, the inside portion of the core will saturate before the outer portion is fully magnetized. Saturation of the inner portion will cause nonlinear current flow which makes pulse shaping more diffficult. In order to maximize utilization of the magnetic material, the core is segmented radially into three sections. The three cores are now driven in parallel and will approach saturation very nearly at the same time. The voltage will, of course, be one-third as high, while the drive current will be three times bigher. An additional benefit of segmenting the core is that the thyratron switch will operate at a more conservative voltage level ( 22 $\mathrm{kV}$ charge), resulting in a more reliable system. A detailed cross-section of the segmented cell is shown in Figure 3.

Each induction core is supported by a mandrel and flanked by a thick plate on one side and a thin one on the other. The thick plate constitutes the ground return and the thin one terminates the high-voltage cable feed. On each side of the core are several layers of Mylar to insulate the high voltage from the ground. The high voltage will be fed through standard high-voltage connectors to the outer core and will be connected to the two inside cores by means of highvoltage wire embedded in the thick plate.

Three cells are bolted together to form a $33 \mathrm{~cm}$ HLP module, and eight modules are assembled together to form a section or cell-block with complete diagnostics capability. Figure 4 shows a complete cell block for the $33 \mathrm{~cm}$ HLP electrostatic focus section with quadrupoles inside. The space between three-cell modules is required for quadrupole support, high voltage feed-through, and articulation devices.

\section{B. Waveform Generator}

The acceleration schedule for ILSE will require careful tailoring of the voltage waveforms at each gap in order to provide energy gain, current multiplication, and longitudinal

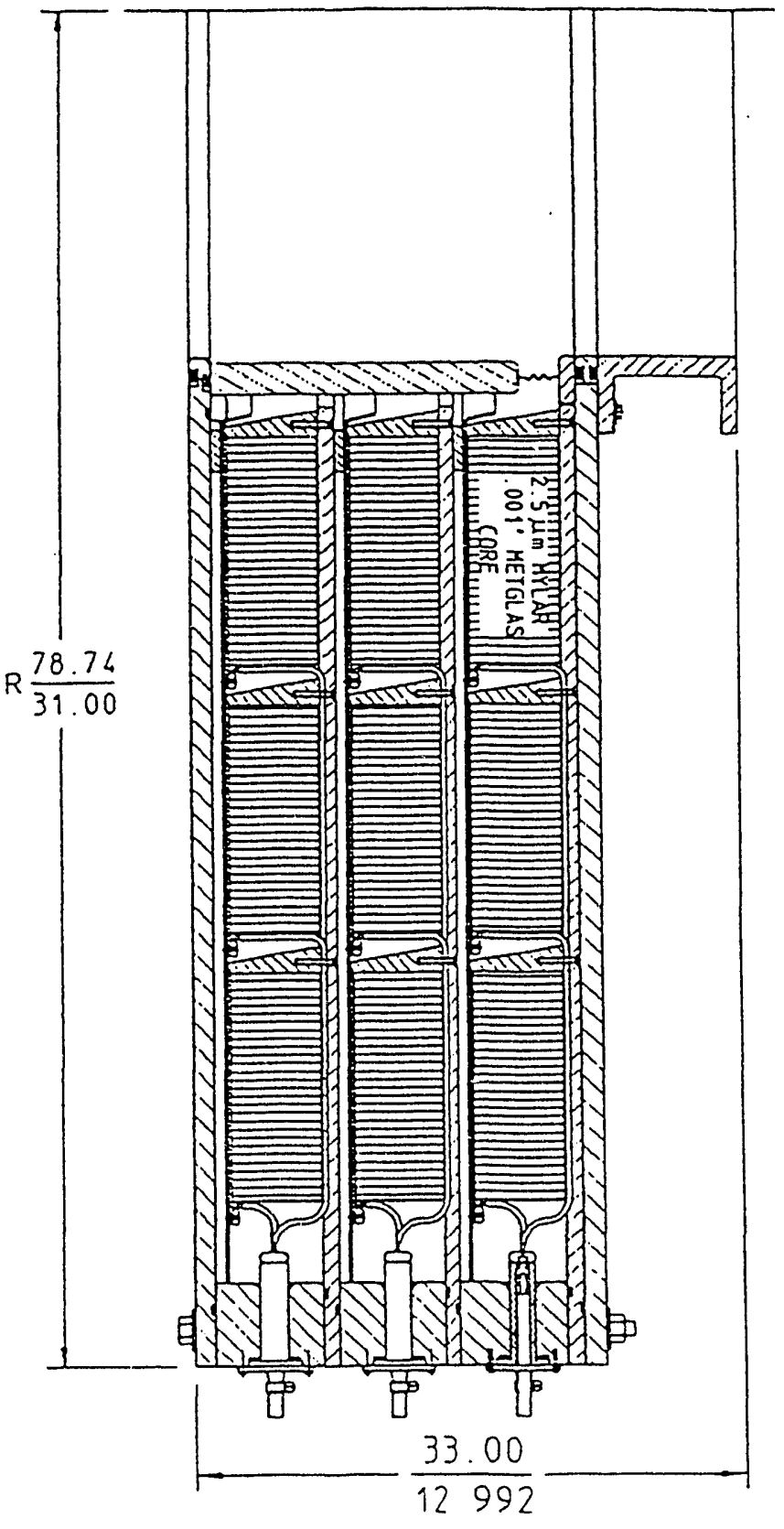

Figure 3. Detailed cross section of radially segmented induction cells for the electrostatic focus section.
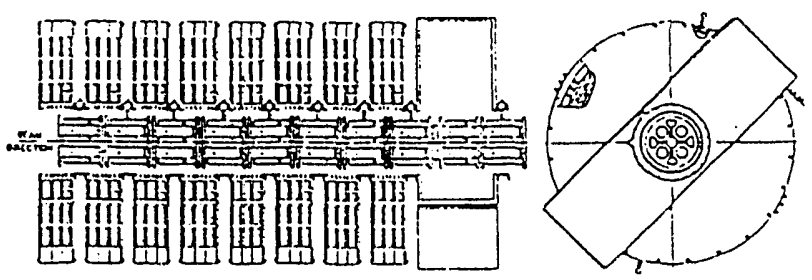

Figure 4. Eight modules of three cells each (33-cm HLP) form a "cell block" in the electrostatic focus section. 
focusing. Figure 5 shows the acceleration waveforms dictated by heavy ion beam transport physics. Initially, the voltage is sawtooth with over $1 \mu$ s duration and then becomes rectangular with shorter and shorter duration. These waveforms should be replicated to within a percent of the ideal. To satisfy these requirements, we have chosen to build pulse-forming networks (PFN) switched by a thyratron. This technology can produce the desired waveforms to within a few percent and will have to be augmented by some active or fast correction devices to meet the specifications. This combination is the most cost-effective way to satisfy all the requirements. Active solid state devices or hard tube modulators to produce all the desired waveforms would be prohibitively expensive. To achieve the desired accuracy, each PFN will be designed with time varying impedance in order to complement the nonlinearity of the induction cell. As can be seen from the magnetizing current, the impedance of the induction cell decreases as the magnetic material approaches saturation; the PFN will likewise have an impedance which "decreases in time." Using this approach, we hope to be within a few percent of the ideal wave shape. The fast correction will then be required to handle a very small fraction of the total current. The voltage to the cell will be slightly higher than required, and the fast correction will basically act as a shunt regulator in a feedback system to lower the voltage to the desired level. The shunt regulator has not been designed yet, but it will consist of a stepdown transformer with FET to act as variable resistors.

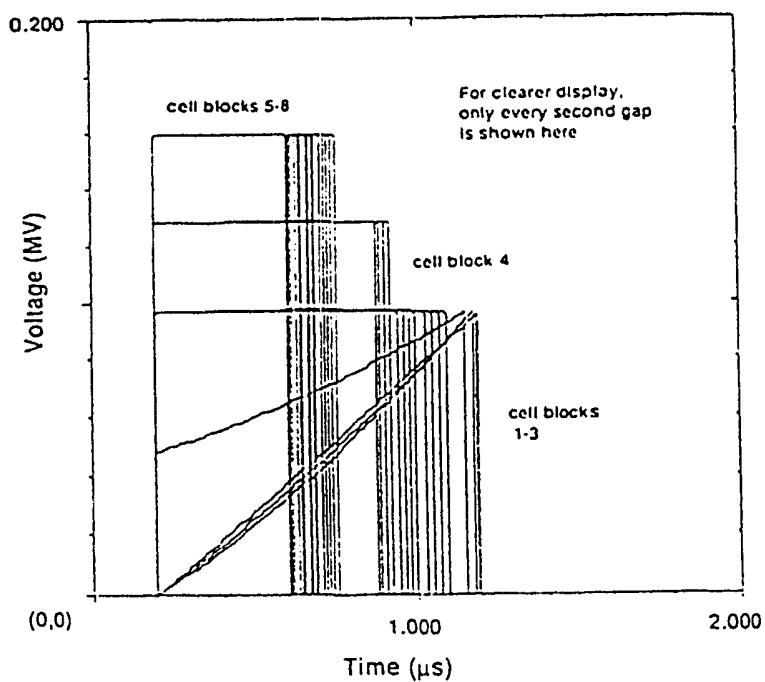

Figure 5. Ideal accelerating waveforms. This figure shows the ideal voltage waveforms for every second accelerating gap needed to achieve self-similar current amplification through the accelerator portion of ILSE.

The block diagram of the complete drive system is shown in Figure 6 . The DC power supply charges the command resonant charger (CRC) which pulse charges the tapered impedance pulse-forming network (PFN) which powers the induction accelerator cores once the thyratron is triggered. During the charge cycle from the CRC to the PFN, the current flows through the cores and partially resets them. Because of their large diameter, however, this charging current is insufficient to fully reset them, and a separate reset pulse is required.

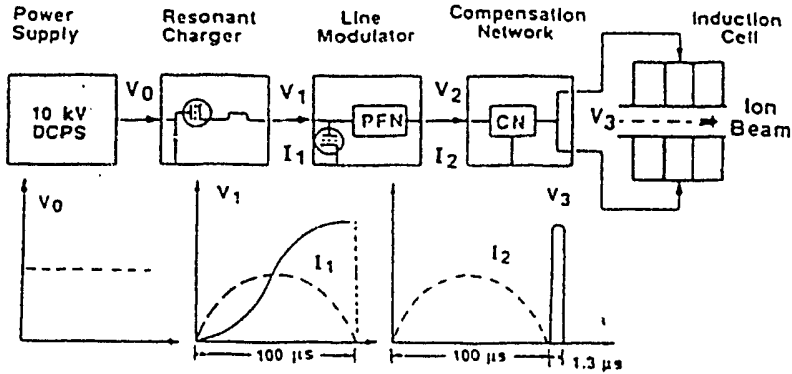

Figure 6. Block diagram of a drive system that generates the acceleration voltage for the induction cells.

The desired sawtooth and rectangular pulses are generated by ladder-type PFNs. These networks use discrete components to build a series of harmonics that together approximate the desired wave shape. Each component or stage of the network is associated with a different portion of the pulse. Taking the lcad impedance as the instantaneous value of the voltage divided by the current for a particular cell, the impedance of each stage can be matched to that load at the time associated with that stage. The two types of networks for generating the sawtooth and rectangular pulses are simulated on the computer prior to building full-scale prototypes. Figure 7 shows a simplified circuit for a rectangular pulse generator with computer simulation and the actual waveform. Figure 8 is the computer simulation for a sawtooth generator.

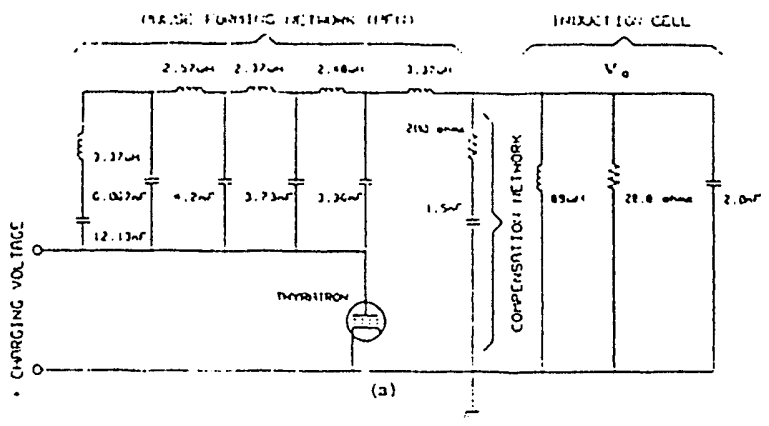

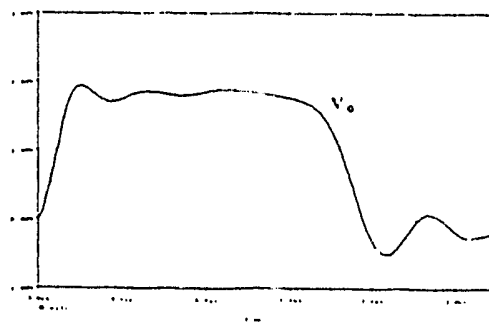

(1)

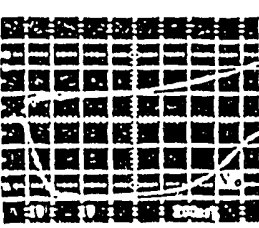

(c)
Figure 7. The schematic, computer-simulation results, and actual test results of the square-wave pulse generator. 

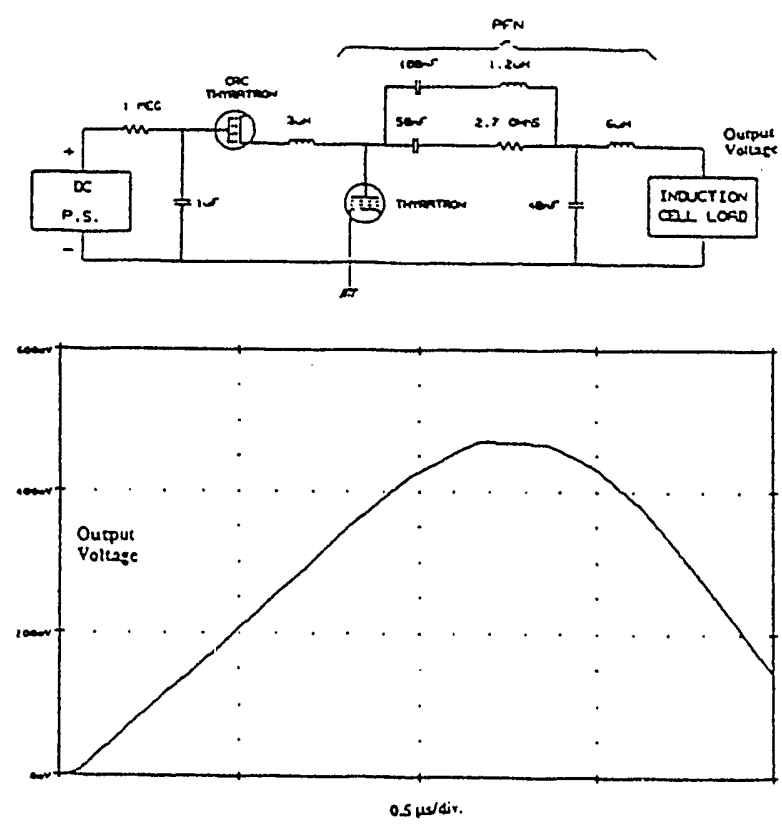

Figure 8. Unipolar network design to generate a ramp, along with the resulting waveform in computer simulation.

\section{Test Stand}

During the past year we have constructed a full scale test stand to study the issues associated with a heavy ion induction accelerator. The test stand consists of three fullscale cells which simulate the $33 \mathrm{~cm}$ HLP module. This module is driven by PFNs which deliver rectangular pulses to the cells. Reset to the cells is provided by a SCR pulser which can drive the magnetic material below $-B_{r}$ to oblain a greater flux swing. The initial cores were all wound using the 2605 S3A material since approximately one tonne was left over from a previous project at a small cost to us. This materials is not the optimum for ILSE, bui it did allow us to assemble the system and develop all the hardware associated with the drive system. We have since tested a variety of other alloys, chosen the optimal material for ILSE, and are currently in the process of replacing the S3A material with SC or other material which will be more suitable. This test stand will eventually house the electrostatic quadrupole and the magnetic quadrupole for in situ testing. The alignment and articulation systems for the quadrupoles will also be prototyped once the project is approved.

\section{SUMMARY}

The HIFAR group at LBL has undertaken the development of an induction accelerator for heavy ion fusion. This accelerator is somewhat unique when compared to the standard ones for electrons in that the waveforms are continually changing throughout the acceleration cycle. This accelerator will address many of the transport issues for driver scale beams and will help resolve many technology issies associated with magnetic materials, waveform generators, alignment, and electrostatic and magnetic quadrupoles. Most importantly, this accelerator will answer many questions associated with the economic viability and reliability of an eventual driver for inertial confinement fusion.

\section{ACFNOWLEDGMENTS}

The technology development for heavy ion induction accelerators presented in this paper reflects the efforts of many individuals in the HIFAR group from the $E E$ and $M E$ departments who support the scientific staff responsible for the physics design of this accelerator. Those individuals who participated in this effort are listed below alphabetically: W. Abraham, D. Berners, I. Broad, B. Dudak, A. Faltens, M. Fong, W. Ghiorso, W. Greenway, R. Hipple, C. Houston, T. Katayanagi, C. Lionberger, H. Meyer, J. Perez, C. Peters, C. Pike, T. Purtell, J. Priyn, J. Rice, J. Stoker, W. Tiffany, D. Vanecek, and from LLNL, F. Deadrick and M. Newton. Their efforts are deeply al preriated.

\section{REFERENCES}

[1] T. Fessenden et al., Proc. 14th Intl. Conf. Plasma Physics and Controlled Nuclear Fusion Research, Germany, September 30 - October 7, 1992.

[2] A. Faltens et al., J. Appl. Phys. 57(1) April 15, 1985.

[3] C.H. Smith, IEEE Trans. Magn. MAG20, 1320 (1984). 


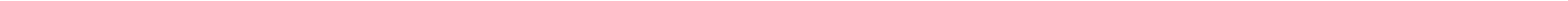




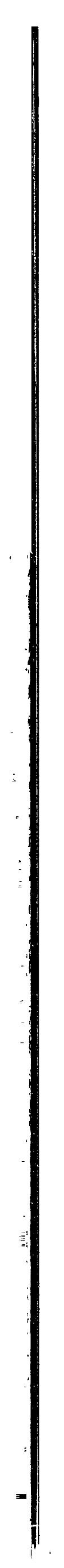

